\title{
Using Wastewater Based Epidemiology as an Upcoming Tool for the Prediction and Control of Covid-19 Disease Outbreaks
}

\author{
Natalie Lowe and Vladmir Bencko* \\ Institute of Hygiene and Epidemiology, 1st Faculty of Medicine, Charles University and the General University Hospital in Prague, Czech Republic \\ *Corresponding author: Vladmir Bencko, Institute of Hygiene and Epidemiology, 1st Faculty of Medicine, Charles University and \\ the General University Hospital in Prague, Czech Republic \\ To Cite This Article: Natalie Lowe and Vladmir Bencko. Using Wastewater Based Epidemiology as an Upcoming Tool for the Prediction and \\ Control of Covid-19 Disease Outbreaks. Am J Biomed Sci \& Res. 2021 - 14(2). AJBSR.MS.ID.001975. DOI: 10.34297/AJBSR.2021.14.001975.
}

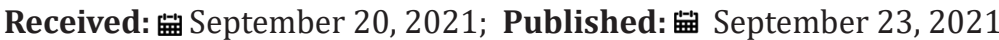

\section{Introduction}

Wastewater provides an excellent source of chemical and biological markers, which in turn serve as tools to decipher human activity. These markers can be analysed to reveal qualitative and quantitative data based on a certain population within a given area. Previous uses of WBE include the monitoring of the use of pharmaceuticals, tobacco, alcohol and illicit drug use within the community however, the implementation for observation of population health is a rapidly expanding field, which has shown immense potential [1]. One of the most renowned uses of WBE was the implementation during the worldwide eradication of Polio. It was invoked to assess the presence of Polio within the community as well as to monitor the efficacy of the vaccination programme. The approach of WBE is based on assumption that any substance that is excreted in the waste of humans is able to be quantified and can be used to back-calculate the original concentration within a specific population, provided the substance is stable in wastewater [2]. One of the greatest advantages of WBE is its capability to reveal the outbreaks at an early stage. Presymptomatic or asymptomatic transmission of SARS-CoV-2 is one of the driving forces behind the rapid spread of the virus, which often goes undetected due to lack of symptoms or display of non-specific symptoms. However, as long as these individuals develop viral shedding via faeces, WBE can detect infected individuals. For example, if an area which previously had a low viral load of SARS-CoV-2 suddenly spikes, it is likely that an outbreak is imminent and decisions can be made to limit the spread of the virus within this given community [3]. The use of WBE for detection of SARS-CoV-2 is based on the principle of viral shedding in stool samples, which is thought to be due to the viral infection of gastrointestinal cells. The viral shedding of SARS-CoV-2, although there is no current consensus, is not considered to be a faecal-oral disease, the viral remnants are inactive and are not able to elicit an infection $[4,5]$. The ability of WBE to include the asymptomatic and oligosymptomatic population means that the evaluated infectivity of the population is almost always underestimated. Studies have shown that in France and Spain, there have been documented concentrations of at least two orders of magnitude higher than the concentrations estimated by other means [4]. Using WBE as a surveillance tool for COVID-19 will allow the spatial display of infection within communities and highlight localisations as 'hotspots'. Individuals living within these 'hotspots' should be tested using RT-PCR, which will give accurate individual results and ensure the early quarantining of individuals, which will reduce or even prevent an outbreak of the disease. Studies have shown that the spread of this virus is heterogenous, meaning it is strongly influenced by local outbreaks $[4,6]$. Using WBE in this way will target the epidemiological characteristics of disease spread, which has the potential to prevent large outbreaks of COVID-19. An example of the application WBE for the early warning of COVID-19 outbreaks was shown by the University of Arizona. Scientists were able to discover high levels of SARS-CoV-2 in the wastewater coming from a student dormitory in August 2020. Using this data, the University was able to take early action and test all the 311 residents of the dormitory using PCR. Clinical testing revealed there were two asymptomatic carriers of the virus, which would have likely caused a significant 
outbreak of the disease when considering the intimate living spaces and concentration of individuals within a student dormitory [3]. WBE also poses solutions to other problems. A number of countries worldwide are struggling due to limited resources and urgency of a clinical testing programme on a country-wide scale [3].

\section{WBE Limitations}

It is important to mention that the quantitative ability of WBE to determine the relationship between levels of viral RNA in sewage and infectivity of the population is dependent on many variables. The main limitation for the estimation of SARS-CoV-2 within the community using WBE is the lack of a reliable stool-shedding rate. In asymptomatic individuals, the rate of viral shedding is typically much lower than in symptomatic patients. In addition to this, there are several variables that affect the shedding rate including the duration of infection, levels of viremia, the age of the patient in addition to the stage and severity of the disease. It can be used combination with clinical testing data when making public health decisions [2,4]. Another deliberation of using WBE for population surveillance of COVID-19 are the ethical considerations. WBE does not focus on individual data, therefore the number of ethical considerations is low when using WBE for viral load. In order to assess the infectivity of a population, ideally smaller sub-populations are monitored so the area of the outbreak can be limited. This raises some ethical considerations as these areas of concern may undergo changed implemented by the public health authority, which in turn can lead to stigmatising behaviours $[2,6,7]$. When considering the potential limitations of WBE for surveillance of SARS-CoV-2 amongst the population, it is important to discuss a number of variables. The viability and confidence interval of WBE relies on the lowest possible prevalence level of viral RNA that can be detected in sewage. This value in turn is determined by a number of variables including the sewage network itself, size of the sampling area, shedding profile of individuals as well as the methods of quantification of the viral RNA and back-calculation models $[3,6,7]$.

Another potential limitation when considering the viability of WBE is the responsiveness to data. Even if the sensitivity of detection is accurate, if the data isn't delivered to and used by authorities in a timely manner then its ineffective. In addition to this, there may be areas where the faecal shedding of SARS-CoV-2 may be consistently detected, these areas require close monitoring for sudden increases in viral RNA remnants in wastewater [8]. The early detection of the presence of SARS-CoV-2 within communities can also give healthcare authorities time to prepare for potential outbreaks, ensuring they have the correct supplies including ventilators, ICU beds and staff available.

\section{Conclusion}

The use of WBE can be applied on a global as well as local scale. The potential for use in the prediction and management of outbreaks is promising. At a relatively low cost and easy implementation into society along with its with minimal ethical considerations, WBE provides a tool, which at this moment in time would contribute to solve many of the problems surrounding early identification of CoVID 19 screening in a local population. WBE method is noninvasive and is done on a population-scale level, so individuals are not targeted, and privacy is respected.

\section{Acknowledgement}

The authors acknowledge support from Research Intention COVMON MV 4 VS BV III

\section{References}

1. Choi P, Tscharke B, Donner E, O’Brien J, Grant S, et al. (2018) Wastewaterbased epidemiology biomarkers: Past, present and future. TrAC Trends in Analytical Chemistry 105: 453-469.

2. Polo D, Quintela-Baluja M, Corbishley A, Jones D, Singer A, et al. (2020) Making waves: Wastewater-based epidemiology for COVID-19 - approaches and challenges for surveillance and prediction. Water Research 186: 116404.

3. Zhu Y, Oishi W, Maruo C, Saito M, Chen R, et al. (2021) Early warning of COVID-19 via wastewater-based epidemiology: potential and bottlenecks. Science of The Total Environment 767: 145124.

4. Gonzalez R, Curtis K, Bivins A, Bibby K, Weir M, et al. (2020) COVID-19 surveillance in Southeastern Virginia using wastewater-based epidemiology. Water Research 186: 116296.

5. Ibrahim N (2020) Epidemiologic surveillance for controlling Covid-19 pandemic: types, challenges and implications. Journal of Infection and Public Health 13(11): 1630-1638.

6. Bivins A, North D, Ahmad A, Ahmed W, Alm E, et al. (2020) WastewaterBased Epidemiology: Global Collaborative to Maximize Contributions in the Fight Against COVID-19. Environmental Science \& Technology 54(13): 7754-7757.

7. Mlejnkova H, Sovova K, Vasickova P, Ocenskova V, Jasikova L, et al. (2020) Preliminary study of Sars-Cov-2 occurrence in wastewater in Czech Republic. Int J Environ Res Public Health 17(15): 5508.

8. Paleologos E, O Kelly B, Tang C, Cornell K, Rodríguez-Chueca J, et al. (2021) Post Covid-19 water and waste water management to protect public health and geoenvironment. Environmental Geotechnics 8(3): 193-207. 